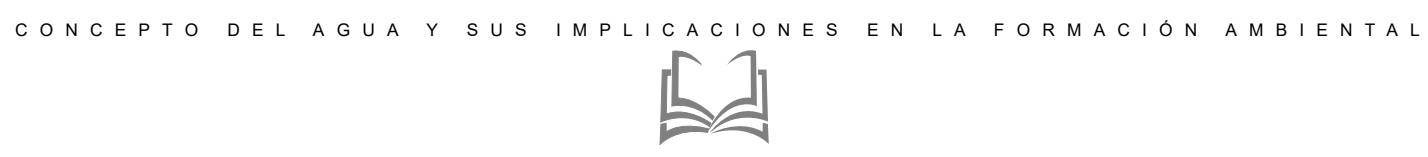

RECIBIDO EL 27 DE OCTUBRE DE 2019 - ACEPTADO EL 28 DE ENERO DE 2020

\title{
CONCEPTO DEL AGUA Y SUS IMPLICACIONES EN LA FORMACIÓN AMBIENTAL
}

\section{CONCEPT OF WATER AND ITS IMPLICATIONS IN ENVIRONMENTAL TRAINING}

\author{
Liliana Caycedo Lozano ${ }^{1}$ \\ Diana Marcela Trujillo Suárez ${ }^{2}$ \\ Grupo de investigación Planificación en \\ Gestión Ambiental Eficiente
}

\section{RESUMEN}

El cuidado de las fuentes hídricas en el territorio

$1 \quad$ Liliana Caycedo Lozano. Colombiana. Magister en docencia de la Química, Licenciada en Química, Docente de tiempo completo de la Universidad Colegio Mayor de Cundinamarca, Programa de Ciencias Básicas. Integrante del grupo de investigación Planificación en Gestión Ambiental Eficiente. Icaycedo@unicolmayor.edu.co.

Número de certificación CVLAC 0000660221

ID ORCID: 0000-0002-9274-3148.

https://scholar.google.es/citations?hl=es\&user=aLW-rxUAAAAJ

2 Diana Marcela Trujillo Suárez. Colombiana. Especialista en Gestión Ambiental, Bacterióloga y Laboratorista Clínico. Integrante del grupo de investigación Planificación en Gestión Ambiental Eficiente. marcet78@gmail.com Número de certificación CVLAC 0001355381 ID ORCID 0000-0001-9462-0339 https://scholar.google.es/citations?hl=es\&user $=m 2 G B T 50 \mathrm{~A}$ AAAJ colombiano es una prioridad que se enmarca dentro de los desafíos que surgen a partir de la propuesta de los Objetivos de Desarrollo Sostenible. En este contexto, es importante el papel que juega la Educación Ambiental en todos los niveles de formación y a partir de ahí se vuelve relevante revisar y aprender los conceptos científicos que se derivan del agua como sustancia pura, lo que implica un trabajo didáctico que tenga en cuenta los diferentes tipos de conceptos que forman parte de las disciplinas científicas que convergen en el lenguaje ambiental.

Por lo tanto, se propone una reflexión en torno a los resultados de dos 
CONCEPTO DEL A GUA Y SUS IMPLICACIONESEN LA FORMACIONAMBIENTAL

investigaciones llevadas a cabo por el grupo de Investigación Planificación en Gestión Ambiental Eficiente de la Universidad Colegio Mayor de Cundinamarca que evidencian la necesidad de la fundamentación conceptual para trascender con argumentos, desde la valoración del agua solo como recurso hídrico hasta la valoración del compuesto como un reactivo con propiedades definitivas para la sostenibilidad del planeta y de nuestro territorio.

PALABRAS CLAVE: educación ambiental, sustancia, agua, compuesto.

\section{ABSTRACT}

The care of water sources in the Colombian territory is a priority that is part of the challenges that arise from the proposal of the Sustainable Development Goals. In this context, the role that Environmental Education plays at all levels of training is important and from there it becomes relevant to review and learn the scientific concepts derived from water as a pure substance, which implies a didactic work that has take into account the different types of concepts that are part of the scientific disciplines that converge in environmental language.

Therefore, a reflection is proposed around the results of two investigations carried out by the Planning Research Group in Efficient Environmental Management of the Universidad Colegio Mayor de Cundinamarca that demonstrate the need for conceptual foundation to transcend arguments, from the valuation of water only as a water resource until the valuation of the compound as a reagent with definitive properties for the sustainability of the planet and our territory.
KEY WORDS: environmental education, substance, water, compound.

\section{SOBRE LOS CONCEPTOS ASOCIADOS AL CONOCIMIENTO CIENTÍFICO}

El trabajo en las aulas gira alrededor de varios procesos de construcción y de descomposición de significados asociados a distintos conceptos pertenecientes a un campo del saber específico, todos estos cambios en representaciones mentales ocurren independientemente de cuál sea la disciplina o ciencia que se esté aprendiendo.

De acuerdo con lo anterior los conceptos se convierten, por un lado, en los protagonistas de los contenidos y por otro, en los indicadores de aprendizaje. La formación en ambiental no es ajena a estos procesos de apropiación y abarca un campo del saber que se construye sobre una gama de conceptos, algunos propios (dependiendo de la especificidad) y otros que son comunes a otras ciencias; pero en los dos casos, con unos significados asociados, por tanto, vale la pena realizar una reflexión en torno a este tema dentro del contexto de la formación ambiental.

Esta convergencia de diferentes ciencias y disciplinas en torno a un discurso ambiental, requiere la comprensión didáctica de la apropiación de los conceptos que se usan en este lenguaje, con el fin de hablar y escribir con conocimiento de causa en un campo todavía novedoso como lo es la educación ambiental.

El sustento argumentativo del saber ambiental se asocia con conceptos, la mayoría de los cuales, se pueden definir como científicos y la mecánica de la apropiación de estos, redundará en la comprensión de lo que se ha venido definiendo como "problemáticas" ambientales.

Proponer una revisión en torno a la esencia de los conceptos científicos y al aprendizaje de los mismos, conlleva necesariamente a lo planteado 
por Jesús Mosterín, en su célebre artículo: "La estructura de los conceptos científicos" publicado en enero de 1978, el cual ha sido de un aporte significativo a la compresión de la racionalidad, ya que con su propuesta éste destacado filósofo, antropólogo y matemático, ha ayudado a entender el cuestionamiento al que llegamos todos los profesores día a día en nuestras aulas: ¿Cómo es que se aprende?, ¿Qué es lo que se aprende?, y, particularmente para aquellos que estamos en el campo de las ciencias naturales el valioso interrogante relacionado con ¿Cómo es que se aprende la ciencia?.

El autor de manera magistral clasifica los conceptos usados en la ciencia en tres tipos y abre un sendero para el trabajo de la didáctica en las ciencias; tales conceptos expuestos corresponden a clasificatorios, comparativos y métricos. (Mosterín, 1978). Los primeros tienen una equivalencia gramatical con sustantivos y adjetivos del lenguaje ordinario, pero principalmente con los primeros, algunos de los cuales pueden ser "demasiado vagos" en la tarea de determinar las cosas a las cuales se aplican; mientras que otros poseen mayor precisión con relación al campo de aplicación.

Los conceptos clasificatorios son básicos e insuficientes tanto para expresar el lenguaje de las ciencias como para comprender la complejidad que estos campos del saber poseen; por esto, surgen los conceptos comparativos, los cuales se relacionan gramaticalmente con los adjetivos, y amplían en argumentación a los clasificatorios, además son un enlace con los conceptos métricos. Al respecto el autor aclara:

"Si identificásemos los conceptos cualitativos con los clasificatorios y los cuantitativos con los métricos, resultaría que en la ciencia se usan otros tipos de conceptos además de los cualitativos y cuantitativos: los conceptos comparativos (o topológicos). Los conceptos comparativos no sólo permiten diferenciar más finamente que los clasificatorios, sino que además representan un primer paso para la posterior introducción de conceptos métricos" (Mosterín, 1978).

Teniendo en cuenta lo anterior, los conceptos métricos se diferencian de los dos tipos anteriores, en primera instancia, porque no tienen una representación gramatical y además porque son "creados" para explicar fenómenos o procesos científicos particulares que pueden ser medibles, es decir para explicar las magnitudes; los conceptos métricos permiten que el vocabulario científico resulte más accequible, pero, no más sencillo ya que en numerosas ocasiones dan lugar a leyes.

La reflexión planteada por Mosterín invita a valorar la importancia que tiene una mejor comprensión de la estructuración conceptual no solo de las ciencias, sino también de las disciplinas y saberes que dan paso a la formación de los profesionales en diferentes campos, como es el caso de lo ambiental.

La didáctica en la formación ambiental en Colombia requiere esa revisión conceptual en atención a que, dentro de los planes de estudio, en los diferentes niveles de formación, se retoman conceptos, o mejor, metaconceptos de otras ciencias y, por tanto, se configura un nuevo lenguaje que necesita precisar y resignificar conceptos de la Química, la Biología, la Física y otros campos del saber.

En este sentido, tiene relevancia lo planteado por (Caycedo, Trujillo, \& García, 2016) con relación a que "El cuidado de la naturaleza como sistema, dependerá en buena parte del conocimiento de las alteraciones fisicoquímicas que en ella sucedan y a partir de las cuales, día a día, se generan grandes cantidades de productos industriales necesarios para el sostenimiento de la economía de los diferentes países, así como niveles importantísimos de energía 
sin los cuales sería imposible concebir la "vida moderna". La minimización en la producción de residuos, es solo una arista de la situación ambiental, en la que todos, como seres sociales, tenemos una gran responsabilidad".

El grupo de Investigación, Planificación en Gestión Ambiental Eficiente de la Universidad Colegio Mayor de Cundinamarca, ha adelantado algunas investigaciones centradas en este aspecto, a partir de una de ellas se puede establecer lo siguiente:

"Es necesario investigar en el campo de la formación ambiental alternativas, desde la didáctica de la Química, que permitan proponer estrategias para la apropiación de conceptos y significados químicos, como: sustancias puras, equilibrios y mecanismos de reacción. De tal forma que, a manera de metaconceptos, acerquen a los futuros profesionales de programas ambientales a un discurso conceptual, metodológico, actitudinal y axiológico que contribuya en la sostenibilidad, más allá del desarrollo sostenible en términos de la preservación de los sistemas naturales" (Caycedo, Trujillo , \& Gómez, 2018).

Los conceptos ambientales, en los diferentes niveles de formación (Básica, Secundaria, y Superior) en Colombia, requieren entenderse como conceptos científicos que están entretejiendo un lenguaje nuevo, pero que no se pueden desligar de la argumentación que dentro de las diferentes disciplinas se ha construido.

A pesar de que se requiera cobertura para el cuidado del ambiente no se puede dejar de lado la connotación de los conceptos que subyacen a las campañas de divulgación; no es solo cuidar por cuidar, se tendrá éxito mayor cuando los niños, los jóvenes y, muy especialmente, los profesionales en áreas ambientales cuenten con los argumentos que subyacen a los conceptos clasificatorios, comparativos y métricos que convergen en este campo del saber.

Recopilando lo anterior, resulta interesante proponer una revisión como metaconcepto ambiental alrededor del significado del "agua", teniendo en cuenta que mucho se sugiere en campañas y en proyectos sobre este recurso, pero vale la pena preguntarse: ¿Qué tanto es interpretado como concepto el agua? Y ¿cuál es el grado de apropiación de los conceptos clasificatorios, comparativos y métricos que giran alrededor de dicha temática? Es probable, que después de ahondar en esto, el cuidado, el ahorro y la valoración del recurso hídrico en Colombia sea más pertinente, responsable e intencionado.

\section{SOBRE EL AGUA COMO CONCEPTO}

En los Objetivos para el Desarrollo Sostenible, la apuesta por la conservación del recurso hídrico se reconoce como esencial para la vida. (Naciones Unidas, 2020). Específicamente, en el ODS No 6 denominado: Agua limpia $y$ saneamiento, se establece que

"El acceso a agua, saneamiento e higiene es un derecho humano, $\mathrm{y}$, sin embargo, miles de millones de personas siguen enfrentándose a diario a enormes dificultades para acceder a los servicios más elementales.

Aproximadamente 1.800 millones de personas en todo el mundo utilizan una fuente de agua potable que está contaminada por restos fecales. Unos 2.400 millones de personas carecen de acceso a servicios básicos de saneamiento, como retretes y letrinas. La escasez de agua afecta a más del $40 \%$ de la población mundial y este porcentaje podría aumentar. Más del $80 \%$ de las aguas residuales resultantes de la actividad humana se vierte en los ríos o 
CONCEPTO DEL AGUA Y SUS IMPLICACIONESEN LA FORMACIONAMBIENTAL

en el mar sin ningún tratamiento, lo que provoca su contaminación" (Naciones Unidas, 2020).

Retomando lo planteado por Naciones Unidas y conectándolo con lo expuesto sobre los conceptos, surge la duda sobre cómo puede y debe actuar la Educación Ambiental y en especial la didáctica, para contribuir en el alcance de las metas que derivan específicamente de este objetivo; metas que se citan a continuación:

"6.1 De aquí a 2030, lograr el acceso universal y equitativo al agua potable a un precio asequible para todos.

6.2 De aquí a 2030, lograr el acceso a servicios de saneamiento e higiene adecuados y equitativos para todos y poner fin a la defecación al aire libre, prestando especial atención a las necesidades de las mujeres y las niñas y las personas en situaciones de vulnerabilidad.

6.3 De aquí a 2030, mejorar la calidad del agua reduciendo la contaminación, eliminando el vertimiento y minimizando la emisión de productos químicos y materiales peligrosos, reduciendo a la mitad el porcentaje de aguas residuales sin tratar y aumentando considerablemente el reciclado y la reutilización sin riesgos a nivel mundial.

6.4 De aquí a 2030, aumentar considerablemente el uso eficiente de los recursos hídricos en todos los sectores y asegurar la sostenibilidad de la extracción y el abastecimiento de agua dulce para hacer frente a la escasez de agua y reducir considerablemente el número de personas que sufren falta de agua.

6.5 De aquí a 2030, implementar la gestión integrada de los recursos hídricos a todos los niveles, incluso mediante la cooperación transfronteriza, según proceda.

6.6 De aquí a 2030, proteger y restablecer los ecosistemas relacionados con el agua, incluidos los bosques, las montañas, los humedales, los ríos, los acuíferos y los lagos.

6.a De aquí a 2030, ampliar la cooperación internacional y el apoyo prestado a los países en desarrollo para la creación de capacidad en actividades y programas relativos al agua y el saneamiento, como los de captación de agua, desalinización, uso eficiente de los recursos hídricos, tratamiento de aguas residuales, reciclado y tecnologías de reutilización

6.b Apoyar y fortalecer la participación de las comunidades locales en la mejora de la gestión del agua y el saneamiento" (Naciones Unidas, 2020).

Apostar por alcanzar las metas descritas implica que los programas en los diferentes niveles de formación dentro del sistema educativo colombiano, expliquen el agua, los significados asociados a ella y las propiedades que este compuesto tiene.

Un ejemplo sencillo: ¿Cómo mejorar la calidad del agua, si no se tiene claro el concepto de solvente universal de este compuesto?; para entender que el agua es el solvente universal, se debe haber construido un significado sobre la solubilidad y qué es la solubilidad?, pues en términos de Mosterín es un concepto que puede definirse en una primera instancia como clasificatorio y comparativo.

Así, la didáctica juega un papel importante en la comprensión del lenguaje ambiental y al desglosar el concepto "agua" se puede llegar a aproximaciones y a construcciones que resignifiquen los "slogan" de las campañas, con el fin de que la trajinada frase "Hay que cuidar 
el agua" tenga una significación argumentada en torno a las propiedades químicas, físicas y biológicas del compuesto. No son razones solo de biodisponibilidad, son argumentos en torno a las propiedades que como molécula covalente polar tiene el agua.

De acuerdo con (Caycedo \& Trujillo, 2019) propiedades como los puentes de hidrógeno, la polaridad molecular y la tensión superficial, entre otras, son características que explican las razones por las cuales el agua es considerada como un recurso natural. Es decir, en los significados de estos conceptos se encuentran algunas de las explicaciones por las cuales hay que cuidar el agua y son dichos conceptos, lo que se deben resignificar y valorar en los cursos que tengan por objeto la apreciación del agua en el marco de la sostenibilidad en Colombia, independientemente del grado de escolaridad.

Los conceptos que subyacen a las propiedades antes mencionadas aluden no solo a clasificaciones y comparaciones, en los términos antes descritos, sino que requieren el uso de conceptos métricos como son polaridad molecular, momento dipolar, enlaces químicos y electronegatividad, entre otros. Atribuyendo al agua significados complementarios, estructurados y más complejos, que pueden a su vez contribuir de fondo a la valoración del recurso y al ahorro de este; es similar a una alfabetización ambiental en torno al "agua" con la rigurosidad que la apropiación de este concepto requiere.

\section{IMPLICACIONES EN LA FORMACIÓN AMBIENTAL}

Bajo las premisas descritas anteriormente, el grupo Planificación en Gestión Ambiental Eficiente, de la Universidad Colegio Mayor de Cundinamarca, ha desarrollado dos proyectos de investigación con el ánimo de indagar en torno a tres categorías de análisis sobre el concepto agua: Actitudinal, aproximación al significado como molécula y aproximación al significado como compuesto.

El primer proyecto se centró en una población de estudiantes que cursaban su formación de Secundaria; mientras que el segundo (Caycedo \& Trujillo, 2019), se llevó a cabo en el ámbito de la educación superior; en los dos casos se aplicó el mismo instrumento estructurado en tres partes, que tenían por objeto indagar sobre las categorías antes descritas.

A partir del análisis y discusión de resultados se pudieron establecer conclusiones muy similares a pesar de la diferencia de las poblaciones (Caycedo \& Trujillo , 2020). La mayoría de los estudiantes, en los dos grupos, muestran una actitud positiva hacia la conservación, el cuidado y el ahorro del recurso hídrico en el territorio nacional; sin embargo, dicha tendencia actitudinal positiva no es argumentada con conocimiento sobre aspectos como la planeación del uso del recurso, la cercanía a las fuentes hídricas y la importancia del agua como constituyente del ecosistema colombiano; esta visión sistémica, requiere la revaloración del recurso con aportes de la base conceptual del mismo, que superen la información superficial y fundamenten el cuidado de las fuentes hídricas con base en el conocimiento.

Figura 1: Representación sistémica de la argumentación del recurso hídrico

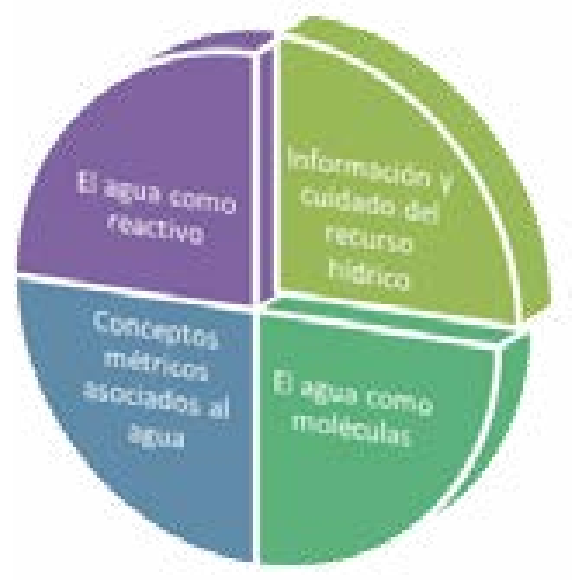


Con relación a la apropiación de los conceptos científicos: tensión superficial, puentes de hidrógeno y polaridad molecular que corresponden, como ya se estableció, a conceptos métricos se encontró que los estudiantes que presentaron la prueba no han construido significados que sirvan como argumentos que aporten al valor del agua desde dichas propiedades.

Por lo anterior, se requiere diseñar estrategias didácticas que trasciendan y que redunden en la apropiación de dichas magnitudes para que el lenguaje ambiental se fundamente en esos significados.

Argumentar la "actitud positiva" hacia el recurso hídrico con el aprendizaje del concepto agua logrará trascender desde la información hacia la formación y desde la representación y el ahorro hacia la comprensión del compuesto como una sustancia pura, que debido a sus propiedades moleculares hace parte de procesos metabólicos que son vitales para la conservación y el equilibrio de los ecosistemas. (Chamizo, 2017), en relación a la pureza de la sustancia, resalta que es necesario precisar sobre el concepto de sustancia y que la pureza depende de la posibilidad técnica de identificar impurezas, por lo cual al indicar la pureza normalmente se menciona la técnica de análisis a través de la cual se reconoció, redefiniéndola junto con el propio conocimiento químico.

Con base en lo anterior, y en el marco del proceso de formación ambiental la reinterpretación del agua como recurso natural precisa asignarle valor conceptual desde diferentes aristas: biológica, química, física, geográfica y económica y con todas ellas es necesario articular un "valor ambiental" que implica la apropiación de los conceptos y significados asociados, ese es uno de los retos de la didáctica ambiental.

Salinas, Covitt \& Gunckel (2013), determinan que es necesario fortalecer un conocimiento integrado basado en pruebas y prácticas científicas para comprender los sistemas socioecológicos, que requieren un examen sobre lo que les da significado.

"El conocimiento disciplinar de la química es obviamente relevante para comprender cómo las sustancias se mezclan con agua, se mueven con ella y se separan, por lo que representa una acumulación que aporta a la discusión pública sobre la calidad y uso de los recursos de agua. Somos críticos de la instrucción científica que no reconoce los recursos cognitivos y los marcos de referencia que los estudiantes han desarrollado para entender el mundo que los rodea".

\section{MARCO LEGAL - LINEAMIENTOS POLÍTICOS, NORMATIVOS YIO LEGALES}

Desde políticas públicas, enmarcadas en leyes, decretos y resoluciones, se establecen las directrices en cuanto a la preservación del medio ambiente, así como se contempla lo relacionado con la educación ambiental; no obstante, es de resaltar la importancia de actualizar dicha normatividad, teniendo en cuenta aspectos de pedagogía y didáctica relacionados con la apropiación de conocimientos y conceptos que, como se ha establecido, prácticamente se convierten en obligatorios para la comprensión desde la formación inicial. 
C ONCEPTO DEL AGUA Y SUS IMPLICACIONES EN LA FORMACION AMBIENTAL

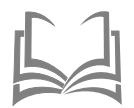

\begin{tabular}{|c|c|}
\hline \multicolumn{2}{|r|}{ NACIONAL } \\
\hline NORMA & OBJETO DE LA NORMA \\
\hline $\begin{array}{l}\text { Constitución } \\
\text { Política de } \\
\text { Colombia }\end{array}$ & $\begin{array}{l}\text { Artículo 67: La educación formará al ciudadano y ciudadana colombianos para } \\
\text { la protección del ambiente. }\end{array}$ \\
\hline Ley 23 de 1973 & $\begin{array}{l}\text { Reglamentada Parcialmente por el Decreto Nacional } 704 \text { de 1986, Reglamentada } \\
\text { Parcialmente por el Decreto Nacional } 305 \text { de 1988, Reglamentada por el Decreto } \\
\text { Nacional } 1974 \text { de } 1989 . \\
\text { Por el cual se conceden facultades extraordinarias al Presidente de la República } \\
\text { para expedir el Código de Recursos Naturales y de Protección al Medio Ambiente } \\
\text { y se dictan otras disposiciones. }\end{array}$ \\
\hline $\begin{array}{l}\text { Decreto } 1337 \text { de } \\
1978\end{array}$ & $\begin{array}{l}\text { Por el cual se reglamentan los artículos } 14 \text { y } 17 \text { del Decreto Ley } 2811 \text { de } \\
\text { 1974. En su artículo No. } 1 \text { establece que el Ministerio de Educación Nacional, } \\
\text { en coordinación con la Comisión Asesora para la Educación Ecológica y del } \\
\text { Ambiente, incluirá en la programación curricular para los niveles pre-escolares, } \\
\text { básicos primarios, básicos secundarios, media vocacional, intermedia } \\
\text { profesional, educación no formal, y educación de adultos, los componentes } \\
\text { sobre ecología, preservación ambiental y recursos naturales renovables. }\end{array}$ \\
\hline Ley 99 de 1993 & $\begin{array}{l}\text { Por la cual se crea el Ministerio del Medio Ambiente, se reordena el Sector } \\
\text { Público encargado de la gestión y conservación del medio ambiente y los } \\
\text { recursos naturales renovables, se organiza el Sistema Nacional Ambiental, } \\
\text { SINA, y se dictan otras disposiciones. }\end{array}$ \\
\hline NORMA & OBJETO DE LA NORMA \\
\hline Ley 115 de 1994 & $\begin{array}{l}\text { Ley General de educación, artículo 5o. fines de la educación. Concordancia } \\
10 \text { y } 12 \text { La adquisición de una conciencia para la conservación, protección y } \\
\text { mejoramiento del medio ambiente, de la calidad de la vida, del uso racional de } \\
\text { los recursos naturales, de la prevención de desastres. }\end{array}$ \\
\hline $\begin{array}{l}\text { Decreto } 1743 \text { de } \\
1994\end{array}$ & $\begin{array}{l}\text { Por el cual se instituye el Proyecto de Educación Ambiental para todos los } \\
\text { niveles de educación formal, se fijan criterios para la promoción de la Educación } \\
\text { Ambiental no formal e informal y se establecen los mecanismos de coordinación } \\
\text { entre el Ministerio de Educación Nacional y el Ministerio del Medio Ambiente. }\end{array}$ \\
\hline $\begin{array}{l}\text { Política Nacional } \\
\text { de Educación } \\
\text { Ambiental } 2002\end{array}$ & $\begin{array}{l}\text { Se aprobó por el Consejo Nacional Ambiental, la Política Nacional de Educación } \\
\text { Ambiental, concertada entre los Ministerios de Educación Nacional y del } \\
\text { Medio Ambiente, llamados así en su momento, lo que significó un gran logro } \\
\text { de coordinación interinstitucional e intersectorial, en el tema de la Educación } \\
\text { Ambiental. Fomenta la importancia de implementar los proyectos ciudadanos } \\
\text { de educación ambiental. }\end{array}$ \\
\hline Ley 1549 de 2012 & $\begin{array}{l}\text { Ley por medio de la cual se fortalece la institucionalización de la política nacional } \\
\text { de educación ambiental y su incorporación efectiva en el desarrollo territorial }\end{array}$ \\
\hline $\begin{array}{l}\text { Decreto } 1075 \text { de } \\
2015\end{array}$ & $\begin{array}{l}\text { Por medio del cual se expide el Decreto Único Reglamentario del Sector } \\
\text { Educación. }\end{array}$ \\
\hline
\end{tabular}


C ONCEPTO DEL AGUA Y SUS IMPLICACIONESEN LA FORMACION AMBIENTAL

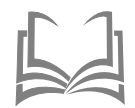

\begin{tabular}{|l|l|}
\hline \multicolumn{2}{|c|}{ INTERNACIONAL } \\
\hline $\begin{array}{l}\text { Conferencia de } \\
\text { Estocolmo }\end{array}$ & $\begin{array}{l}\text { Primera conferencia de las Naciones Unidas sobre medio ambiente. Consagra el } \\
\text { derecho del hombre a vivir en un medio de calidad y proteger y mejorar el medio } \\
\text { para las generaciones futuras, además resalta la importancia de la educación } \\
\text { ambiental. }\end{array}$ \\
\hline $\begin{array}{l}\text { Conferencia de } \\
\text { Tbilisi }\end{array}$ & Primera conferencia de las Naciones Unidas sobre educación ambiental. \\
\hline \multicolumn{1}{|c|}{ NORMA } & \multicolumn{1}{c|}{ OBJETO DE LA NORMA } \\
\hline $\begin{array}{l}\text { Comisión mundial } \\
\text { sobre medio } \\
\text { ambiente y } \\
\text { desarrollo }\end{array}$ & $\begin{array}{l}\text { Se trabajó el tema de desarrollo sostenible, como base fundamental del proceso } \\
\text { de concertación entre la protección del medio ambiente y el desarrollo. }\end{array}$ \\
\hline
\end{tabular}

Fuente: adaptado de Cristancho, G. \& Trujillo, D. (2018). Apropiación de conceptos ambientales para la comunidad del humedal de techo Bogotá - Cundinamarca, mediante el diseño de una estrategia pedagógica (trabajo de grado especialización). Fundación Universitaria del Área Andina, Bogotá,

Colombia.

\section{CONCLUSIONES}

La formación ambiental en Colombia, en los diferentes niveles de educación, requiere un aporte desde la didáctica que se centre en la revisión y apropiación conceptual del nuevo lenguaje que se configura, desde diferentes campos del saber tales como la Química, la Biología y la Física, entre otros.

Los conceptos ambientales, desde la didáctica, requieren entenderse y apropiarse como conceptos científicos que se entretejen, pero que no se pueden desligar de la argumentación que dentro de las diferentes disciplinas se les ha asignado y representado.

Es preciso resignificar y valorar las explicaciones conceptuales por las cuales hay que cuidar el agua y esta reflexión didáctica debe redundar en los cursos que tengan por objeto la apreciación del recurso hídrico, en el marco de la sostenibilidad en Colombia, independientemente del grado de escolaridad.

A partir de la formación ambiental, la reinterpretación del agua como recurso natural requiere asignarle valor conceptual desde diferentes aristas con el fin de articular un "valor ambiental" que implique la apropiación de conceptos y significados interdisciplinares e interrelacionados, ese es uno de los retos de la didáctica ambiental.

En Colombia, se requiere actualizar las directrices normativas en Educación Ambiental de manera que se posibilite trascender, desde lo informativo hacia lo formativo, valorando y reinterpretando nuestros recursos naturales con un lenguaje propio pero fundamentado desde lo conceptual. 


\section{REFERENCIAS BIBLIOGRÁFICAS}

Chamizo, J. (2017). La cuarta revolución química (1945-1966). De las sustancias a las especies químicas, Educación Química, 8(4), (págs. 202210). https://doi.org/10.1016/j.eq.2017.07.001. En http://www.sciencedirect.com/science/article/ pii/S0187893X1730071X

Caycedo, L., \& Trujillo, D. (2020). Análisis del significado sobre el concepto agua en un grupo de estudiantes universitarios. En proceso de Publicación . En U. C. Cundinamarca, Diario de Campo. Sello Editorial. Universidad Colegio Mayor de Cundinamarca.

Caycedo, L., \& Trujillo, D. (2019).

Categorización de significados del concepto agua en estudiantes de educación media . En U. C. Cundinamarca, Diario de Campo. En proceso de publicación. Bogotá: Sello Editorial. Universidad Colegio Mayor de Cundinamarca.

Caycedo, L., Trujillo , D., \& Gómez, J. (2018). Diagnóstico comparativo del uso del metaconcepto "compuesto" por estudiantes de programas ambientales en las Instituciones de Educación Superior IES Colombianas . En U. C. Cundinamarca, Diario de Campo. La experiencia: Requisito para la visibilidad, la divulgación y el impacto de la Investigación (págs. 132-154). Bogotá : Sello Editorial Universidad Colegio Mayor de Cundinamarca.

Caycedo, L., Trujillo, D., \& García, S. (2016). La responsabilidad social, un componente esencial de la formación en un programa de química ambiental. Misión Jurídica, 223-232.
Mosterín, J. (1978). La Estructura de los conceptos científicos. Investigación y Ciencia, 82-93.

Naciones Unidas. (22 de 06 de 2020). Objetivos de dearrollo sostenible . Obtenido de un.org/sustainabledevelopment/es/ development-agenda/

Salinas, I., Covitt, B., Gunckel, K. (2013). Sustancias en el agua: progresiones de aprendizaje para diseñar intervenciones curriculares, Educación Química, 24(4), (págs. 391-398), https://doi.org/10.1016/ S0187-893X(13)72492-3.En http://www. sciencedirect.com/science/article/pii/ S0187893X13724923) 